\title{
Arrangements of Transition-Metal Atoms in Three Types of Al-Co-Ni Decagonal Quasicrystals Studied by Cs-Corrected HAADF-STEM
}

\author{
K. Hiraga ${ }^{1}$ and A. Yasuhara ${ }^{2}$ \\ ${ }^{1}$ Institute for Materials Research, Tohoku University, Sendai 980-8577, Japan \\ ${ }^{2}$ EM Application Group, EM Business Unit, JEOL Ltd., Tokyo 196-8558, Japan
}

\begin{abstract}
Three types of $\mathrm{Al}-\mathrm{Co}-\mathrm{Ni}$ decagonal quasicrystals in $\mathrm{Al}_{72.5} \mathrm{Co}_{11} \mathrm{Ni}_{16.5}, \mathrm{Al}_{71} \mathrm{Co}_{14.5} \mathrm{Ni}_{14.5}$ and $\mathrm{Al}_{71.5} \mathrm{Co}_{16} \mathrm{Ni}_{12.5}$ alloys have been examined by Cs-corrected high-angle annular detector dark-field scanning transmission electron microscopy (HAADF-STEM), which has an enough resolution to represent individual TM atoms as bright dots in observed images of decagonal quasicrystals. On the basis of the structure of a $\mathrm{W}$ (AlNiCo) crystalline approximant, transition-metal (TM) atoms and mixed sites (MSs) of Al and TM atoms are separately distinguished in HAADF-STEM images. Most of TM atoms are arranged with pentagonal tilings of a bond-length of $0.76 \mathrm{~nm}$, and MSs are located inside of pentagonal frames with definite orientations. All of the TM atoms and MSs are located at lattice points of a Penrose lattice of an edge-length of $0.25 \mathrm{~nm}$, and arranged with a bond-orientational order. The present result shows that the structures of Al-Co-Ni decagonal quasicrystals should be characterized as $\mathrm{BOO}$ arrangements of transition-metal atoms, instead of previous models of BOO arrangements of $2 \mathrm{~nm}$ atom clusters. [doi:10.2320/matertrans.M2012340]
\end{abstract}

(Received October 12, 2012; Accepted January 18, 2013; Published March 25, 2013)

Keywords: quasicrystal, decagonal quasicrystal, structure, aluminum-cobalt-nickel, aberration-corrected scanning transmission electron microscopy, high-angle annular detector dark-field scanning transmission electron microscopy, stem, haadf-stem

\section{Introduction}

Six types of decagonal quasicrystals have been found in Al-Co-Ni alloys, ${ }^{1-5)}$ and their structures have been interpreted by quasiperiodic arrangements of columnar atom clusters with a bond-orientational order (BOO) that is formed by a constant length of bonds connecting atoms or atom clusters and definite orientations of bonds related to decagonal symmetry. ${ }^{6,7)}$ The structures of five types of the Al-Ni-Co decagonal quasicrystals, except for one referred to as Ni-rich basic structure, can be characterized by ordered arrangements of two types of the clusters with different orientations of pentagonal symmetry, and can be divided into two groups, e.g. structures formed with arrangements of one type of clusters with the same orientations and those of ordered arrangements of the two types of the clusters with different orientations. ${ }^{6,7)}$ Recent Cs-corrected STEM has an enough resolution to represent individual TM atoms as separated bright dots in observed images of decagonal quasicrystals. ${ }^{8,9)}$ Therefore, in the present paper, we intended to reexamine the structures of the $\mathrm{Al}-\mathrm{Co}-\mathrm{Ni}$ decagonal quasicrystals by Cs-corrected HAADF-STEM observations.

Before we started the present study, we observed HAADFSTEM images of a crystalline phase of W-(AlCoNi) ${ }^{9)}$ which is considered to be an important approximant for understanding the structures of the Al-Co-Ni decagonal quasicrystals, ${ }^{10)}$ and we examined the correspondence between the observed HAADF-STEM images and its structure. That result shows that arrangements of transition-metal (TM) atoms and mixed sites (MSs) of $\mathrm{Al}$ and $\mathrm{TM}$ atoms can be directly determined from those of separated bright dots in HAADFSTEM images. From the knowledge, we intend to understand the structures of the three decagonal quasicrystals as arrangements of TM atoms by Cs-corrected HAADF-STEM. The three decagonal quasicrystals can be understood as structures of two quasiperiodic planes with a period of $0.4 \mathrm{~nm}$ along the periodic axis, if weak diffuse reflections showing a period of $0.8 \mathrm{~nm}$ are neglected. $\left.{ }^{6}\right)$ Therefore, the structures of the decagonal quasicrystals are characterized by arrangements of TM atoms and MSs in the two quasiperiodic planes.

\section{Experimental Procedures}

Alloys of nominal compositions of $\mathrm{Al}_{72.5} \mathrm{Co}_{11} \mathrm{Ni}_{16.5}$, $\mathrm{Al}_{71} \mathrm{Co}_{14.5} \mathrm{Ni}_{14.5}$ and $\mathrm{Al}_{71.5} \mathrm{Co}_{16} \mathrm{Ni}_{12.5}$ were prepared by melting high-purity (99.99\%) $\mathrm{Al}, \mathrm{Co}$ and $\mathrm{Ni}$ metals in an arc furnace in an $\mathrm{Ar}$ atmosphere. Parts of those $\mathrm{Al}_{72.5} \mathrm{Co}_{11^{-}}$ $\mathrm{Ni}_{16.5}, \mathrm{Al}_{71} \mathrm{Co}_{14.5} \mathrm{Ni}_{14.5}$ and $\mathrm{Al}_{71.5} \mathrm{Co}_{16} \mathrm{Ni}_{12.5}$ ingots were sealed in evacuated quartz tubes, annealed at $900^{\circ} \mathrm{C}$ for $120 \mathrm{~h}$, at $1000^{\circ} \mathrm{C}$ for $65 \mathrm{~h}$ and $900^{\circ} \mathrm{C}$ for $72 \mathrm{~h}$, respectively, and then quenched in water. HAADF-STEM images were taken with the incident beam parallel to the periodic axis by using of a Cs-corrected scanning electron microscope (JEMARM200F).

\section{Experimental Result and Discussion}

The structure of a decagonal quasicrystal in the $\mathrm{Al}_{71^{-}}$ $\mathrm{Co}_{14.5} \mathrm{Ni}_{14.5}$ alloy has been characterized as an ordered arrangement of two kinds of columnar clusters with different orientations of decagonal symmetry. ${ }^{11)}$ The fundamental lattice, which is constructed by connecting the clusters, is a rhombic lattice with a bond-length of $2 \mathrm{~nm}$. The ordered arrangement of the two kinds of the clusters has been interpreted by a CsCl-type body-centered hypercubic lattice in a five-dimensional space. ${ }^{12)}$

Figure 1 shows HAADF-STEM images of the $\mathrm{Al}_{71} \mathrm{Co}_{14.5^{-}}$ $\mathrm{Ni}_{14.5}$ decagonal quasicrystal. In the image of Fig. 1(a), one can see two kinds of clusters, which are constructed by ten bright dots surrounding a pentagonal arrangement of bright dots, with different orientations of central pentagons, as indicated by solid and dashed circles. The clusters are connected by bonds of a length of $2 \mathrm{~nm}$ and form a rhombic lattice. The clusters connected by the bonds have different 

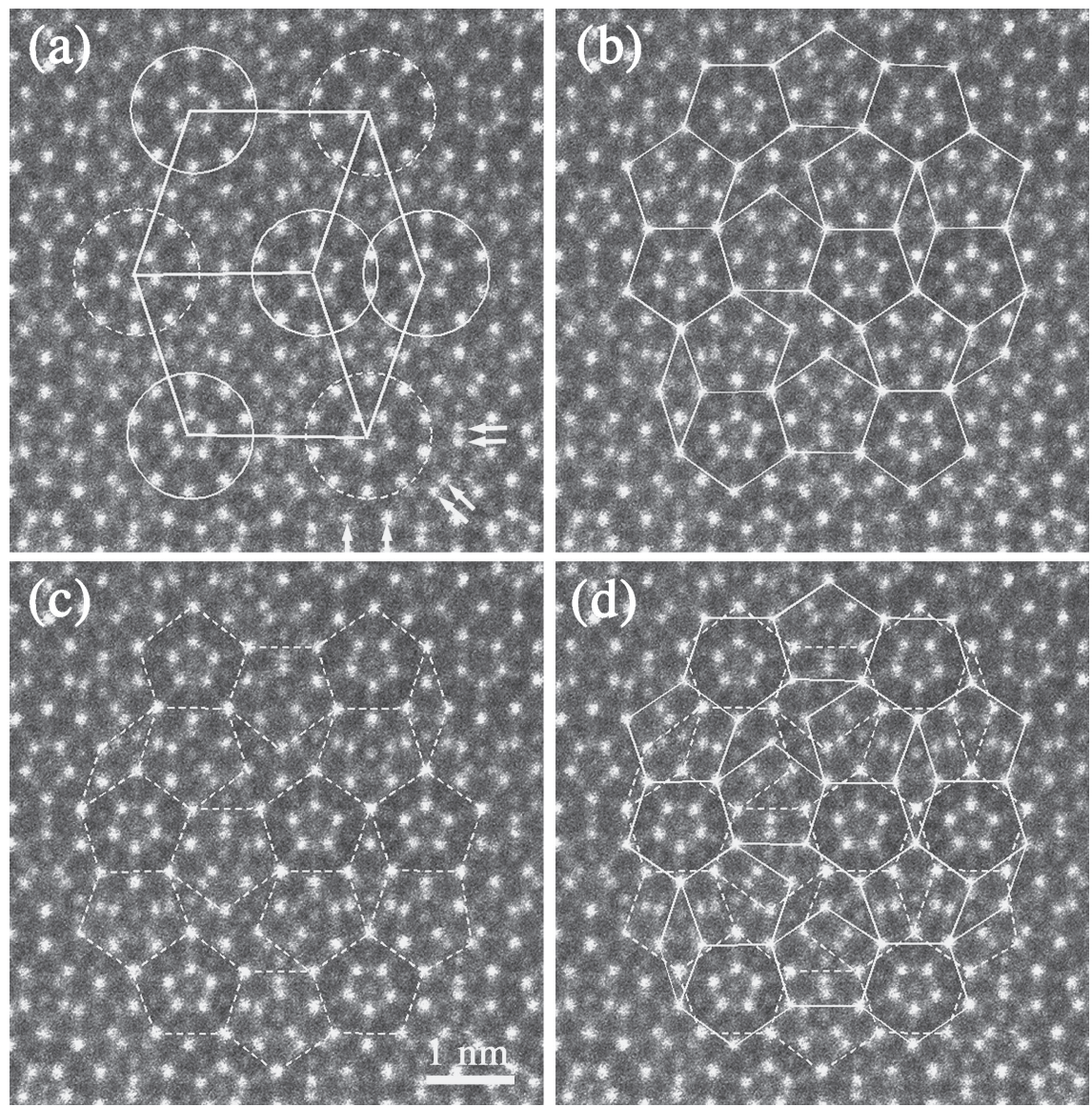

Fig. 1 HAADF-STEM images of the $\mathrm{Al}_{71} \mathrm{Co}_{14.5} \mathrm{Ni}_{14.5}$ decagonal quasicrystal. In (a), one can see two kinds of clusters, which are constructed by ten bright dots surrounding a pentagonal arrangement of bright dots, with different orientations of central pentagons, as indicated by solid and dashed circles. The clusters are connected by bonds of a length of $2 \mathrm{~nm}$ and form a rhombic tiling. Most of bright dots in the image can be connected by lines with a length of $0.76 \mathrm{~nm}$, as shown in (b) and (c). Note double pentagonal distributions of bright dots inside of upward pentagonal frames in (b) and downward ones in (c), and pentagonal arrangements of bright dots inside of downward pentagonal frames in (b) and upward ones in (c). Almost all of bright dots in the image can be interpreted by bright dots connected by pentagonal tilings of solid and dashed lines and those in double pentagonal disributions in (b) and (c), as can be seen in (d).

orientations of central pentagons. However, it should be noted that bright dots in a concentric circle outside the cluster indicated by circles have no decagonal and pentagonal symmetry, as can be seen from an arrangement of two bright dots and single ones indicated by pairs of arrows and single ones, respectively. That is, the arrangement of bright dots in the HAADF-STEM image shows no existence of $2 \mathrm{~nm}$ atom clusters with pentagonal symmetry.

Most of bright dots in the HAADF-STEM image can be connected by lines with a bond-length of $0.76 \mathrm{~nm}$, as shown in Figs. 1(b) and 1(c). The structure of the decagonal quasicrystal is formed by two planes of A and B stacking along the periodic axis, and so the bright dots connected by the solid and dashed lines correspond to TM atoms on the A and B planes, respectively. In Figs. 1(b) and 1(c), one can see double pentagonal distributions of bright dots, which are composed with pairs of bright dots along the directions of fivefold rotational symmetry, in upward pentagonal frames in Fig. 1(b) and downward ones in Fig. 1(c), whereas in the downward pentagonal frames in Fig. 1(b) and upward ones in Fig. 1(c), pentagonal distributions of bright dots are placed. The five dots of most outside of the double pentagonal distribution correspond to TM atoms and/or MSs on a different plane from the tiling plane, and the other (a)
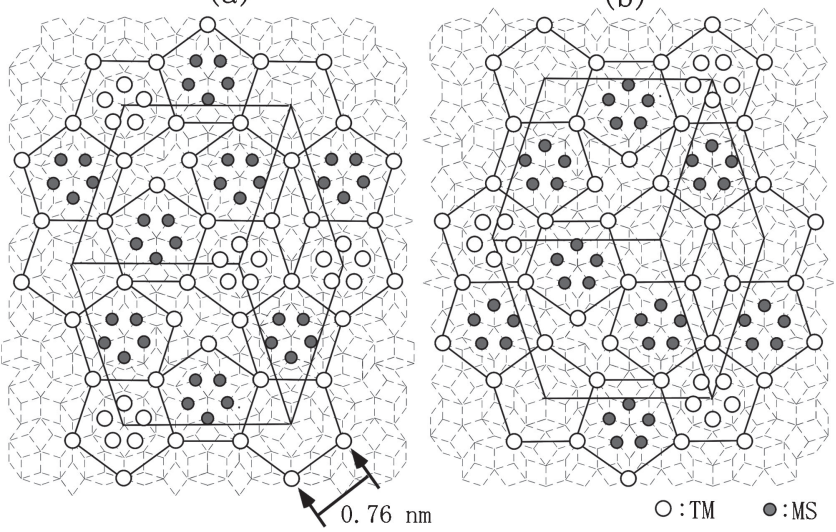

Fig. 2 Arrangements of TM atoms and MSs on the A (a) and B (b) planes of the $\mathrm{Al}_{71} \mathrm{Co}_{14.5} \mathrm{Ni}_{14.5}$ decagonal quasicrystal, derived from Fig. 1. The weak dashed lines indicate a Penrose lattice of an edge-length of $0.25 \mathrm{~nm}$.

inside five dots correspond to MSs on the tiling plane, as can be seen in Fig. 1(d). This structural feature is nearly the same as that in the structure of the W-(AlCoNi) crystalline phase. ${ }^{10)}$

Figure 2 shows arrangements of TM atoms and MSs on the $\mathrm{A}$ and $\mathrm{B}$ planes, derived from the HAADF-STEM image of Fig. 1. All of TM atoms and MSs are located at lattice 

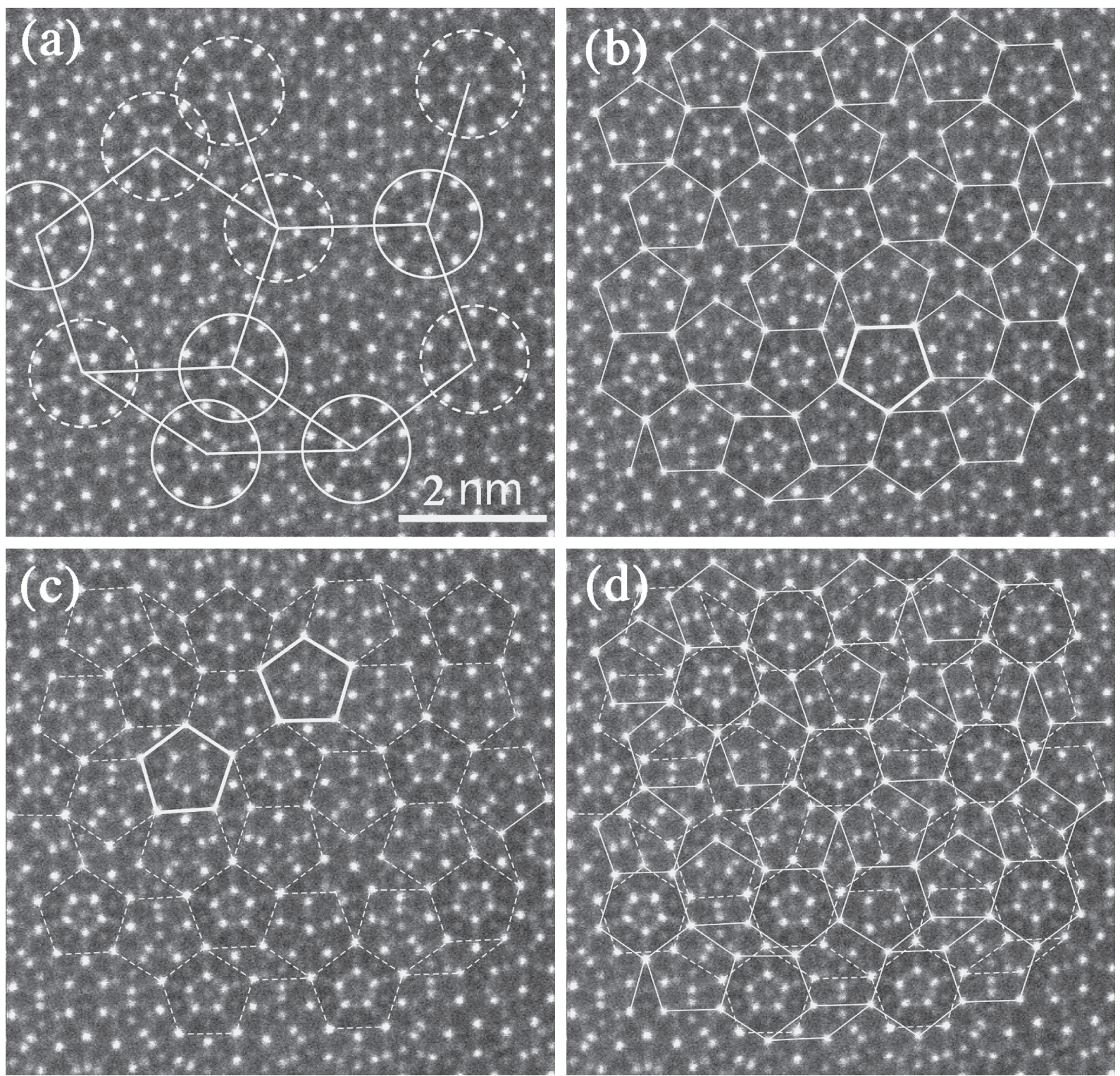

Fig. 3 HAADF-STEM images of the $\mathrm{Al}_{71.5} \mathrm{Co}_{16} \mathrm{Ni}_{12.5}$ decagonal quasicrystal. In (a), one can see an arrangement of two kinds of clusters with different orientations of central pentagons, indicated by solid and dashed circles, in a pentagonal tiling with an edge-length of $2 \mathrm{~nm}$. Bright dots in the image can be connected by lines with a length of $0.76 \mathrm{~nm}$, as shown in (b) and (c), and double pentagonal and pentagonal distributions of bright dots are located in pentagonal frames with definite orientations, like that of Fig. 1. The bright dots with asymmetry in pentagons drawn by thick lines in (b) and (c) can be explained by the projection of bright dots connected by pentagonal tiling and those in double pentagonal distributions on the other plane, as can be seen in (d).

points of a Penrose lattice with an edge-length of $0.25 \mathrm{~nm}$, and consequently they are arranged with BOO. A similar distribution of the MSs, which are always located in upward pentagonal frames in Fig. 2(a) and downward ones in Fig. 2(b), can be seen in the structure of the B plane of the W-(AlCoNi) crystalline phase. ${ }^{9,10)}$ It should be noticed that the pentagonal tilings of Fig. 2 include ship-shaped polygons with three acute-angle triangles in addition to pentagons and thin rhombuses.

Figure 3 is HAADF-STEM images of the $\mathrm{Al}_{71.5} \mathrm{Co}_{16} \mathrm{Ni}_{12.5}$ decagonal quasicrystal. The decagonal quasicrystal is fundamentally formed by a pentagonal tiling of two types of clusters with a bond-length of $2 \mathrm{~nm},{ }^{13)}$ and so ordering that two clusters connected with a bond of $2 \mathrm{~nm}$ are always different types of clusters, is broken in pentagonal tiles, as can be in Fig. 3(a). In the same way as Fig. 1, bright dots are located at lattice points in pentagonal lattices with a bondlength of $0.76 \mathrm{~nm}$, as can be seen in Figs. 3(b) and 3(c). Also, in all of upward pentagonal frames in Fig. 3(b) and downward ones in Fig. 3(b), double pentagonal distributions of bright dots are placed. On the other hand, in most of downward pentagonal frames in Fig. 3(b) and upward ones in Fig. 3(b), one can see pentagonal distributions of bright dots, which are located at lattice points of the pentagonal lattice of a $2 \mathrm{~nm}$ bond-length, and a few pentagonal frames indicated by thick solid lines have asymmetric distributions of bright dots. The bright dots in the asymmetric arrangements correspond to those connected by a pentagonal tiling in another plane, as can be seen in Fig. 3(d). Arrangements of $\mathrm{TM}$ atoms and MSs in the A and B planes can be directly determined from the image of Fig. 3, as shown in Fig. 4. In the pentagonal tilings of Fig. 4 , one can see the appearance 


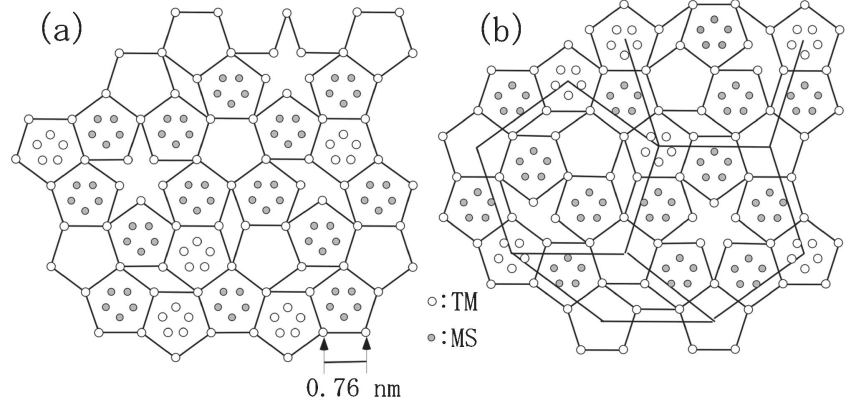

Fig. 4 Arrangements of TM atoms and MSs on the A (a) and B (b) planes of the $\mathrm{Al}_{71.5} \mathrm{Co}_{16} \mathrm{Ni}_{12.5}$ decagonal quasicrystal, derived from Fig. 3. of star-shaped polygons in addition to pentagons, thin rhombuses and ship-shaped polygons.

Figure 5 shows HAADF-STEM images of the $\mathrm{Al}_{72.5} \mathrm{Co}_{11^{-}}$ $\mathrm{Ni}_{16.5}$ decagonal quasicrystal. In the image, an area including a pentagon, squashed hexagon and deformed polygon with an edge-length of $2 \mathrm{~nm}$ is shown. As the same as the $\mathrm{Al}_{71} \mathrm{Co}_{14.5} \mathrm{Ni}_{14.5}$ and $\mathrm{Al}_{71.5} \mathrm{Co}_{16} \mathrm{Ni}_{12.5}$ decagonal quasicrystals of Figs. 1 and 3, bright dots can be connected by two pentagonal lattices with an edge-length of $0.76 \mathrm{~nm}$ and all of downward pentagonal frames in Fig. 5(b) and upward ones in Fig. 5(c) have double pentagonal distributions of bright dots. On the other hand, in upward pentagonal frames in Fig. 5(b) and downward ones in Fig. 5(c), one can see
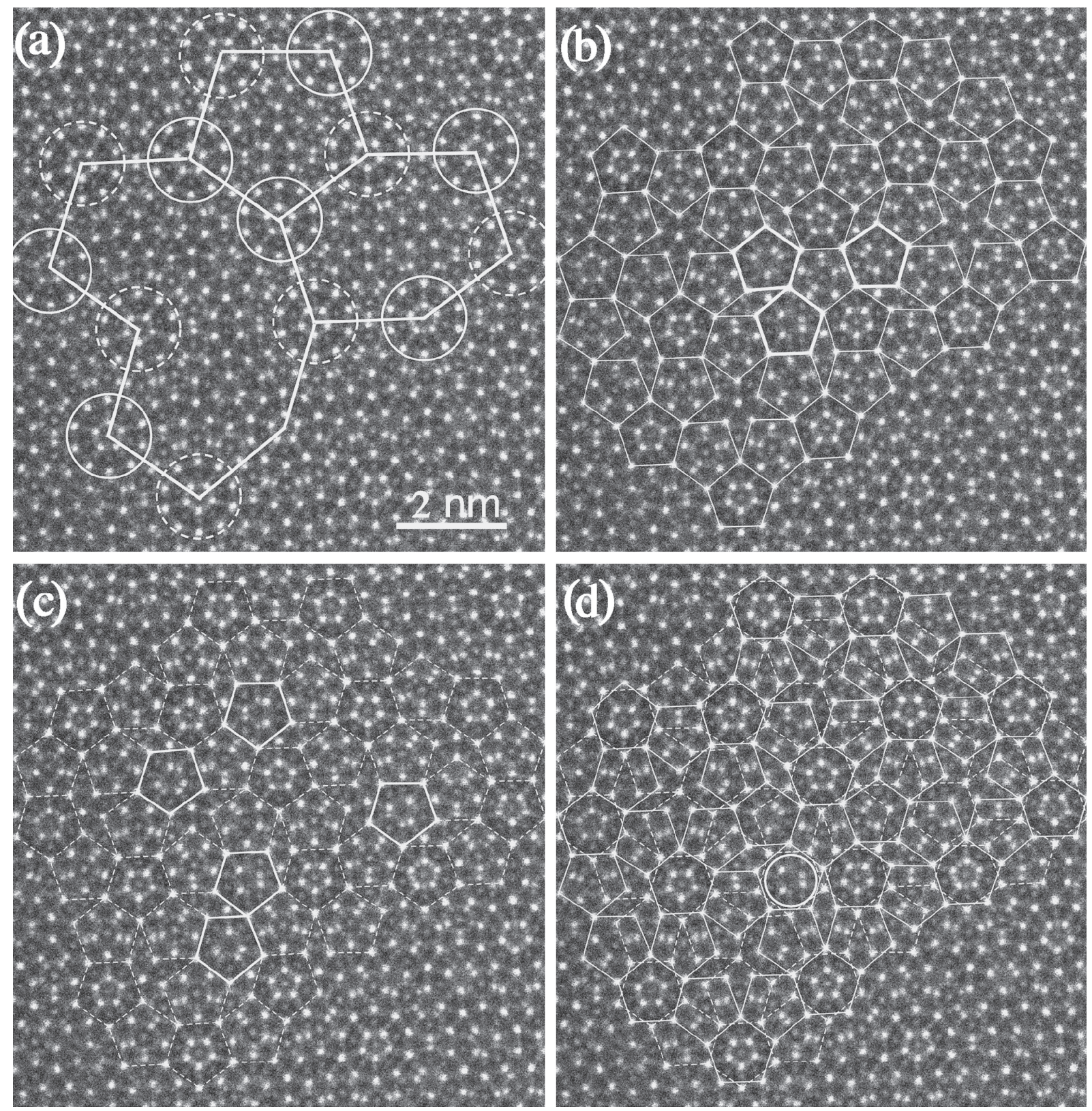

Fig. 5 HAADF-STEM images of the $\mathrm{Al}_{72}{ }_{5} \mathrm{Co}_{11} \mathrm{Ni}_{16.5}$ decagonal quasicrystal. In (a), one can see an arrangement of two kinds of clusters with different orientations of central pentagons, indicated by solid and dashed circles. Bright dots in the image can be connected by lines with a length of $0.76 \mathrm{~nm}$, as shown in (b) and (c), and double pentagonal and pentagonal distributions of bright dots are located in pentagonal frames with definite orientations, like that of Fig. 1. The bright dots with asymmetric arrangements in pentagons drawn by thick lines in (b) and (c) can be explained by the projection of bright dots connected by pentagonal tiling and those in double pentagonal distributions on the other plane, as can be seen in (d), except for those in a circle in (d). 


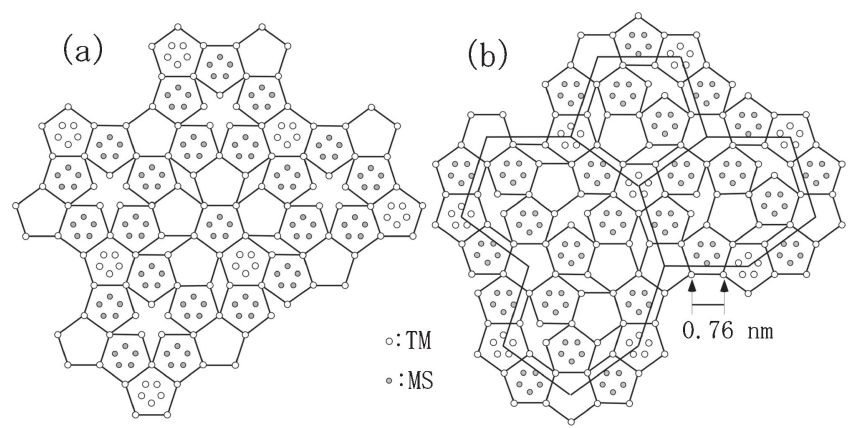

Fig. 6 Arrangements of TM atoms and MSs on the A (a) and B (b) planes of the $\mathrm{Al}_{72.5} \mathrm{Co}_{11} \mathrm{Ni}_{16.5}$ decagonal quasicrystal, derived from Fig. 5.

pentagonal arrangements of bright dots, which are located at the centers of the atom clusters in Fig. 5(a), and asymmetric distributions of bright dots, as can be seen in pentagons indicated by thick lines in Figs. 5(b) and 5(c). Most of the bright dots arranged with asymmetry can be interpreted as those connected by a pentagonal tiling or those in the double pentagonal distributions of bright dots on the other plane, as can be seen in Fig. 5(d). However, the bright dots in a circle in Fig. 5(d) can not be explained by the above interpretation, but they can be interpreted as TM atoms and/or MSs connected by pentagonal tiling with an edge-length of 0.56 $(=0.76 / \tau) \mathrm{nm}$.

Figure 6 shows arrangements of TM atoms and MSs in the $\mathrm{A}$ and $\mathrm{B}$ planes, derived from the HAADF-STEM image of Fig. 5 and the similarity of the structure of the W-(AlCoNi) approximant. The tilings of arrangements of TM atoms is formed by pentagons, thin rhombuses, ship-shaped polygons and star-shaped polygons.

\section{Summary}

The structures of three types of $\mathrm{Al}-\mathrm{Co}-\mathrm{Ni}$ decagonal quasicrystals with two quasiperiodic planes in $\mathrm{Al}_{72.5} \mathrm{Co}_{11^{-}}$ $\mathrm{Ni}_{16.5}, \mathrm{Al}_{71} \mathrm{Co}_{14.5} \mathrm{Ni}_{14.5}$ and $\mathrm{Al}_{71.5} \mathrm{Co}_{16} \mathrm{Ni}_{12.5}$ alloys have been examined by Cs-corrected HAADF-STEM, which has an enough resolution to represent individual TM atoms as separated bright dots in observed images of decagonal quasicrystals. On the basis of the structure of the W(AlNiCo) crystalline approximant, TM atoms and MSs can be distinguished from HAADF-STEM images. Most of TM atoms are arranged with pentagonal tilings of an edge-length of $0.76 \mathrm{~nm}$, and MSs are located inside of pentagonal frames with definite orientations. All of the TM atoms and MSs are located at lattice points of a Penrose lattice of an edge-length of $0.25 \mathrm{~nm}$, and arranged with BOO. The present result shows that the structures of Al-Co-Ni decagonal quasicrystals must be interpreted by $\mathrm{BOO}$ arrangements of TM atoms, instead of previous structure models of decagonal atom clusters with $2 \mathrm{~nm}$ in diameter. ${ }^{7)}$

\section{REFERENCES}

1) A. P. Tsai, A. Inoue and T. Masumoto: Mater. Trans. JIM 30 (1989) 150-154.

2) K. Hiraga, F. J. Lincoln and W. Sun: Mater. Trans. JIM 32 (1991) 308314.

3) K. Edagawa, M. Ichihara, K. Suzuki and S. Takeuchi: Philos. Mag. Lett. 66 (1992) 19-25.

4) A. P. Tsai, A. Fujiwara, A. Inoue and T. Masumoto: Philos. Mag. Lett. 74 (1996) 233-240.

5) S. Ritsch, C. Beeli, H.-U. Nissen, T. Godecke, M. Scheffer and R. Luck: Philos. Mag. Lett. 78 (1998) 67-75.

6) K. Hiraga, T. Ohsuna, W. Sun and K. Sugiyama: Mater. Trans. 42 (2001) 2354-2367.

7) K. Hiraga: Advances in imaging and electron physics, vol. 122 (2002) (Elsevier Science (USA)) pp. 1-86.

8) E. Abe: Scanning Transmission Electron Microscopy, imaging and Analysis (Edited by S. J. Pennycook and P. D. Nellist, Springer New York Dordrecht Heidelberg London) pp. 583-614.

9) A. Yasuhara, K. Saito and K. Hiraga: Proc. of Aperiodic (2012), in press.

10) K. Sugiyama, S. Nishimura and K. Hiraga: J. Alloy. Compd. 342 (2002) 65-71.

11) K. Hiraga, T. Ohsuna, S. Nishimura and M. Kawasaki: Philos. Mag. Lett. 81 (2001) 109-115.

12) K. Hiraga, T. Ohsuna and W. Sun: Mater. Sci. Eng. A 312 (2001) 1-8.

13) K. Hiraga, T. Ohsuna and S. Nishimura: Mater. Trans. 42 (2001) 10811084. 